\title{
Factors Affecting Eluation Characteristics of Sorption Generators of Technetium-99m
}

\author{
V. S. Skuridin ${ }^{1}$, V. I. Chernov ${ }^{1,2}$, V. L. Sadkin ${ }^{1}$, E. S. Stasyuk ${ }^{1}$, \\ N. V. Varlamova ${ }^{1}$, A. S. Rogov ${ }^{1, a)}$, E. A. Nesterov ${ }^{1}$, E. A. Ilina ${ }^{1}$, \\ L. A. Larionova ${ }^{1}$, and A. A. Medvedeva ${ }^{1,2}$ \\ ${ }^{1}$ Institute of Physics and Technology, Tomsk Polytechnic University, Tomsk, 634050 Russia \\ ${ }^{2}$ Tomsk Cancer Research Institute, Tomsk, 634050 Russia

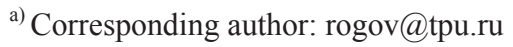

\begin{abstract}
The influence of the adsorbed mass of molybdenum on the width of eluation profiles of generators and the patterns of molybdenum distribution in the amount of chromatographic columns by scanning them on the germaniumgallium detector using collimating device are studied. The boundary conditions under which the maximum value of ${ }^{99 \mathrm{~m}} \mathrm{Tc}$ yield from generators $Y_{\mathrm{e}}=1$ are defined. After scanning the columns, it was found out that the degree of filling the columns with molybdenum $\mathrm{Q}$ and the value of its maximum adsorption depend naturally on the total weight of the adsorbed mass. In order to achieve the condition $Y_{\mathrm{e}}=1$ the value of $Q$ should be at least $85 \%$.
\end{abstract}

\section{INTRODUCTION}

Short-lived isotope technetium-99m is widely used for highly informative diagnostic studies in various fields of medicine. Currently, more than $80 \%$ of all radionuclide studies are performed using radiopharmaceuticals based on it $[1,2]$. To obtain the isotope technetium-99m installations called technetium generators are used.

Chromatographic generators are most widely used. World production of chromatographic (sorption) generators of technetium-99m is based on the use of highly active ${ }^{99} \mathrm{Mo}(200 \mathrm{Ci} / \mathrm{g})$ isolated from the fission products of uranium-235, which causes significant technical and environmental problems. An alternative to uranium technology is organization of ${ }^{99}$ Mo production by the reaction of radiative capture $(n, \gamma)$ using enriched molybdenum 98 at research reactors widespread in Russia and in the world. This technology has virtually no waste, but the resulting product has a low specific activity of ${ }^{99} \mathrm{Mo}$ at $6-10 \mathrm{Cu} / \mathrm{g}$.

The use of such low active materials having a large amount of the carrier in the form of stable nuclei of molybdenum for the production of generators requires the use of columns of increased size, which increases eluation profile of generators and as a consequence, to a decrease in the volume activity of eluted preparation of technetium$99 \mathrm{~m}$.

The aim of the work was to investigate the factors influencing the eluation characteristics of generators of technetium-99m, manufactured by neutron irradiation of enriched molybdenum oxide-98 [3, 4].

\section{MATERIALS AND METHODS}

The study of patterns of change in eluation yield of technetium-99m, depending on the adsorbed mass of molybdenum-98 irradiated by neutrons, was carried out on generators " $99 \mathrm{~m}$ Tc-GT-TOM" produced in TPU.

Elution of technetium- $99 \mathrm{~m}$ from generators in the form of sodium pertechnetate solution was carried out by pumping of $0.9 \%$ solution of $\mathrm{NaCl}$ (eluent) in portions of $\sim 2 \mathrm{ml}$ into $10 \mathrm{ml}$ vials through columns followed by measuring the activity of ${ }^{99 \mathrm{~m}} \mathrm{Tc}$ on the radiometer RLG-07-c. 


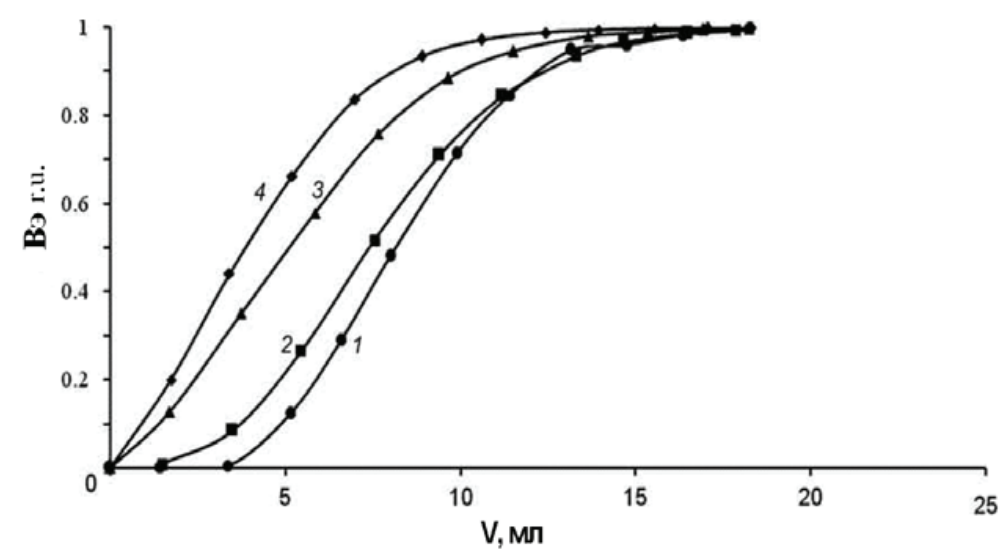

FIGURE 1. Change of eluation yield of ${ }^{99 \mathrm{~m}} \mathrm{Tc}$ depending on the volume of saline for generators with different adsorbed mass of molybdenum: 0.070 (1), 0.085 (2), 0.1366 (3), $0.177 \mathrm{~g}(4)$

Based on these results, the dependence of changes in the yield of ${ }^{99 m} \mathrm{Tc}\left(Y_{\mathrm{e}}\right)$ on the volume of passed eluent (eluation profiles of generators), as well as dependence of changes in eluation yield on the adsorbed mass of molybdenum-98 for different volumes of the eluent are developed.

Changes in the activity of adsorbed ${ }^{99} \mathrm{Mo}$ along the length of chromatographic column were determined by single-channel pulse-height analyzer Strahlungsmessgerat 20046 (Germany) by scanning the columns above the detector with collimator. The calculations of the distribution of inactive molybdenum-carrier in the generator columns were made as the results of these measurements using the ratio where $m_{i}$ and $A_{i}$ - the mass of molybdenum and activity of ${ }^{99}$ Mo on the $i$ th sector of the column:

$$
m_{i}=A_{i} \frac{m_{\mathrm{Mo}}}{\Sigma A},
$$

$\Sigma A$ - total activity of ${ }^{99}$ Mo in columns, $m_{\mathrm{Mo}}$ - total adsorbed mass of molybdenum.

\section{EXPERIMENTAL RESULTS AND DISCUSSION}

Chromatographic column filled with aluminum oxide (90 activated, acidic $(0.063-0.200 \mathrm{~mm})$ used for chromatography, "Merck") were used for research. Before application of various amounts of molybdenum in the form of the solution of sodium polymolybdate on the generator columns, aluminum oxide was treated with the $0.05 \mathrm{M}$ solution of hydrochloric acid, in an amount of $3.8 \times 10^{-4}$ mole per $1 \mathrm{~g}$ of oxide. The acid treatment was carried out to create active centers of adsorption (AC) in the oxide. Then various amounts of molybdenum in the form of the solution of sodium polymolybdate were applied on generator columns. In the next step, elution of generators with certain portions of eluant was carried out and the resulting activity of each portion was measured. Yield value (rel. units) was calculated as the ratio of the activity of ${ }^{99 \mathrm{~m}} \mathrm{Tc}$ eluted from the generator in the volume $V_{i}$, to its total activity, resulting at full elution. Dependencies developed based on the results of these calculations are shown in Fig. 1.

They show that with an increase molybdenum mass eluation profile of generator becomes narrower. For example, for the complete elution of ${ }^{99 \mathrm{~m}} \mathrm{Tc}$ from generator with adsorbed weight of $0.070 \mathrm{~g}$ it is necessary to pass $16-17 \mathrm{ml}$ of eluent through its column and $8-9 \mathrm{ml}$ will be enough at a weight of $0.177 \mathrm{~g}$. Accordingly, even at the same total activity of ${ }^{99 \mathrm{~m}} \mathrm{Tc}$ eluted from generators the volumetric activity of the preparation will be about 2 times higher in the latter case than in the first.

In this regard, given eluation curves lead to the calculation of the mass of the adsorbed molybdenum, ensuring the narrowest profile of generator for any given conditions of preparation of aluminum oxide, as well as to the prediction of optimum volume of eluent that could be recommended by the radiologist for maximum volume activity in the performance of different studies. 


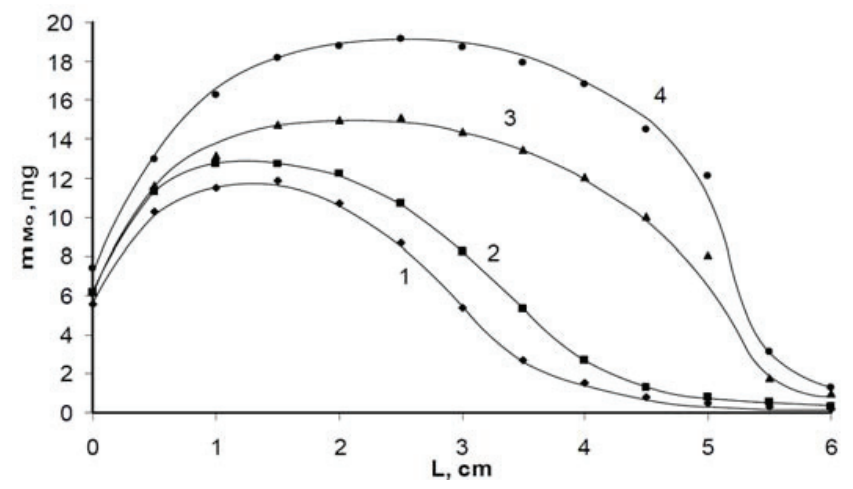

FIGURE 2. Distribution of Mo in generator columns depending on the adsorbed mass $m_{\mathrm{Mo}}$ : 0.070 (1), $0.085(2), 0.1366(3), 0.177 \mathrm{~g}(4)$

To clarify the reasons for the delay of low out washing of ${ }^{99 \mathrm{~m}} \mathrm{Tc}$ at low adsorbed mass of molybdenum patterns of its distribution in the generator columns was studied by scanning them above the detector with collimator. Calculation of the adsorbed amounts of molybdenum mi on separate sectors of the column was performed using Eq. (1). Figure 2 shows the corresponding dependences of $m_{i}$ on $L$.

It can be seen from dependencies in Fig. 2 that in all cases in the first half of the columns (at their input) a maximal adsorption value $G_{\max }$ is reached (maximum concentration), after which a decrease is observed. The values were calculated by the average value of the sum of three maximum values $m_{\max }=\sum m_{i} / 3$ and is divided into the mass of oxide, attributable to the section of the column. Furthermore, dependencies in Fig. 2 show that there are areas not filled with molybdenum on the yield of the columns, and hence containing a large number of vacant active centers in the aluminum oxide, where "inhibition" of ${ }^{99 \mathrm{~m}} \mathrm{Tc}$ is possible during the process of its elution from the column. The greater the size of these sections is, the smaller the total adsorbed mass is. The dependence of the degree of filling of the column with molybdenum $Q_{i}$ on its adsorbed mass is shown in Fig. 3. Here, for the calculation of values $Q_{i}$ the following ratio is used

$$
Q_{i}=m_{\mathrm{Mo}} / G_{\max } m_{O x} .
$$

Figure 4 shows the dependence of the change of the yield of $Y_{\mathrm{e}}$ on $Q_{i}$ for the $8.5 \mathrm{ml}$ of eluent solution. It is described by the equation:

$$
Y_{e}=1.141+0.871 \ln O_{i}
$$

from which it follows that in order to achieve the maximum value of yield $=1$ the degree of filling of the column with molybdenum should be 0.85 , i.e., $85 \%$. In accordance with the dependence in Fig. 3 this limit value of $Q_{i}$ is reached during the adsorption of more than $0.20 \mathrm{~g}$ of molybdenum.

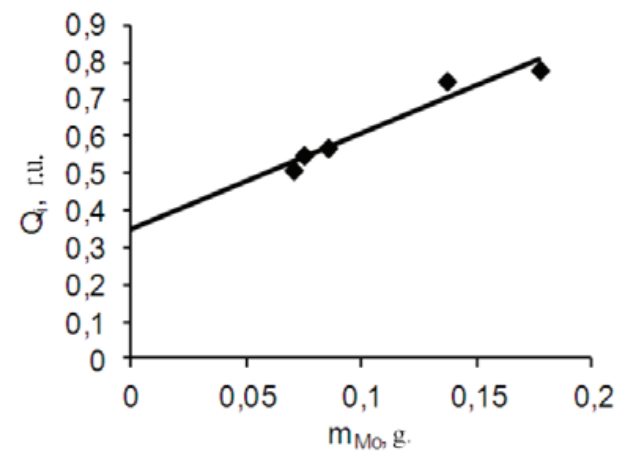

FIGURE 3. Change of the degree of filling of columns $Q_{i}$ depending on the mass of the adsorbed molybdenum $m_{\mathrm{Mo}}$

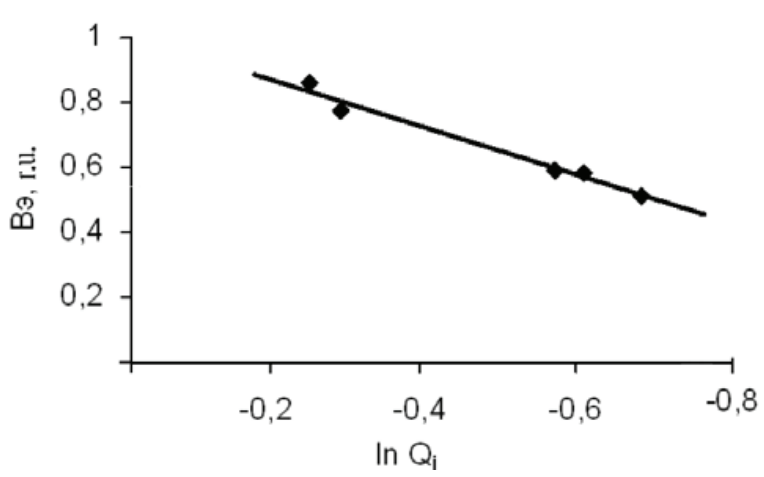

FIGURE 4. Change of the yield $Y_{\mathrm{e}}$ depending on $\ln Q_{i}$ for the volume $8.5 \mathrm{ml}$ 


\section{CONCLUSION}

The study of eluation characteristics of technetium- $99 \mathrm{~m}$ generators, manufactured based on molybdenum-98 irradiated with neutrons, and the study of distribution of molybdenum in the generator columns show.

The width of eluation profile of generators depends on the adsorbed mass of molybdenum. At the same time, with its increase the profile of generators becomes narrower, reaching a minimum at a certain limiting mass corresponding to the maximum sorption capacities of the sorbent-aluminum oxide.

The value of maximum adsorption of molybdenum and the degree to which the columns are filled with it depend on the total input mass. It is shown that to achieve the yield of ${ }^{99 \mathrm{~m}} \mathrm{Tc}$ equal to 1 from generator, the degree of filling of the columns should be $85 \%$. A possible reason for the observed decrease in yield at low filling of the columns is "inhibition" of ${ }^{99 \mathrm{~m}} \mathrm{Tc}$ on the vacant active centers of aluminum oxide.

The studies allow concluding that during the pre-sorption preparation of aluminum oxide the number of active centers created in them at acidizing must comply with specified (based on the specific activity of ${ }^{99} \mathrm{Mo}$ ) values of adsorbed mass of molybdenum required for the manufacture of generators with the required consumer ratings.

\section{ACKNOWLEDGMENTS}

This work was financially supported by the Russian Ministry of Education and Science (RFMEFI57514X0034).

The study reported in this article was conducted according to accepted ethical guidelines involving research in humans and/or animals and was approved by an appropriate institution or national research organization.

The study is compliant with the ethical standards as currently outlined in the Declaration of Helsinki.

All individual participants discussed in this study, or for whom any identifying information or image has been presented, have freely given their informed written consent for such information and/or image to be included in the published article.

\section{REFERENCES}

1. S. Webb, Physics of Imaging in Medicine, V. 1 (Mir, Moscow, 1991), pp. 190-318.

2. G. E. Kodina and V. N. Korsunskiy, Status and process of use of technetium-99m radiopharmaceuticals in Russia, Radiochemistry 38(5), 385-388 (1997).

3. V. S. Skuridin, E. S. Stasyuk, E. A. Nesterov, V. L. Sadkin, and A. S. Rogov, Adsorption of ${ }^{99 \mathrm{~m}}$ Tc on Aluminum Oxide, Radiochemistry 53(5), 529-533 (2011).

4. V. S. Skuridin, V. L. Sadkin, E. S. Stasyuk, E. A. Nesterov, and A. S. Rogov, A procedure for sorbent pretreatment for the production of high-activity ${ }^{99} \mathrm{Mo} /{ }^{99 \mathrm{~m}} \mathrm{Tc}$-generators based on enriched ${ }^{98} \mathrm{Mo}$, Radiochemistry 54(4), 391-394 (2012). 